\title{
A rotating fiber precipitating apparatus and its application
}

\author{
Shi ling ${ }^{1, a}$, liu xuexin ${ }^{3}$, Yu xinming ${ }^{1}$, Xiong jiming ${ }^{3}$, Li wen ${ }^{3}$
}

1 Hubei Key Laboratory of Industrial Fume \& Dust Pollution Control, Hubei Wuhan 430056; 2 School of chemistry and environmental engineering, Jianghan University, Hubei Wuhan 430056; 3

Hubei Smile New Material Co., Ltd, Hubei Hongan ,438400

aling_s_h@163.com,

Keywords: filtering, rotating fiber, wet precipitator, precipitator

Abstract. Experimental investigation is conducted for a new type precipitating apparatus, and results are reported, meantime its application also are reported. Application and experimental results show that the collection efficiency for respirable dust is above $80 \%$ and $92 \%$, under application and experiment condition, respectively, that the pressure loss of the rotating fiber apparatus is less than $200 \mathrm{~Pa}$.

\section{Introduction}

Dust generating is inevitable product of drilling, blasting, excavating, minerals transporting and exhausted gas from internal-combustion engine in tunnel. Dust is dispersed into the mine atmosphere the ventilating air flow, and travels downwind. Despite extensive research into the development of dust control techniques, it is still difficult for the control of dust in tunnel, especially, the respirable dust. Because the respirable dust can result to the Pneumoconiosis, study extensively and deeply on the respirable dust control technology have obtained some valuable achievements. There are several principally dust control technology: ventilation, spray, dust cleaning $^{[1]}$.

Though dust precipitating method that has been developed is able to realized dust pollutants control in principle, such as cyclones and scrubbers which is effective for large-size particle removal, the interest that the existed technology may obtain is getting less for the removal of fine particles. Particles of size of 1 micron or smaller are to be removed used fibrous filter, but the humidity in tunnel is very high, the space is narrow relatively, the application of classic fibers filtering encounters limit with the jam of the filter ${ }^{[2]}$. The application of the ESP also is limited due to its special requirement of resistant corrosion ${ }^{[3]}$. Wet dust collector is focused by researchers at home and abroad, due to this precipitator's structure is simple and reliable operating. Qian and $\mathrm{Li}$ have designed the wet dust collector according to the atmosphere feature of the mine tunnel, some properties of the collector applied in tunnel are tested ${ }^{[4,5]}$.

It is distinct feature that fiber filter efficiency is high and pressure loss is also high, and it is clogged easily under high humidity condition. The droplet may be dust collector due to the inertia collision and interception mainly, and the coagulating also play a part. it will be a promise dust 
cleaning method, if two of fiber filter and wet combine, some defects can be conquered.

According to above thinking, A rotating fiber dust collecting apparatus is constructed, a serials of experimental are conducted, and results are reported. Next,a wet precipitator is designed used the rotating fiber as a key part, this precipitator is used under mineral tunnel condition, some testing are conducted.

\section{mechanism rotating fiber}

The principle of rotating fiber is shown in Fig.1. Fiber is served as dust collector, four are basic mechanism are involved classically: interception, inertial impaction, and diffusion, at the same time that the dust-loaded airstream is cut due to fiber rotating is considered. The parameters of the rotating fiber apparatus is referenced [6] detail.

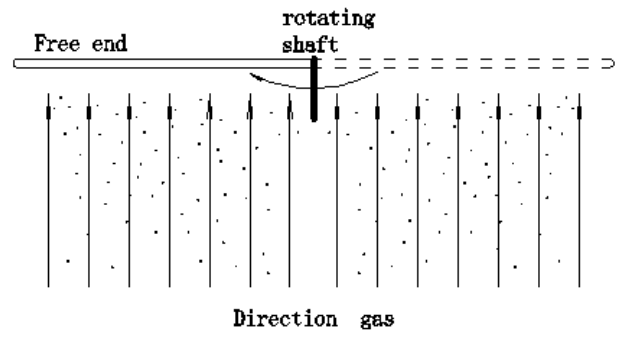

a

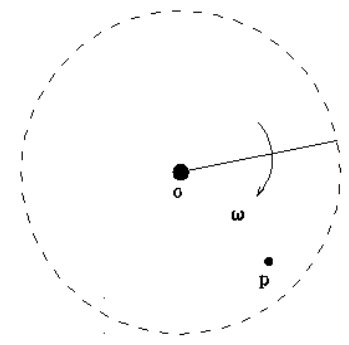

b

a Front view b Top view

Fig. 1 Principle of rotating fiber

The process of removal of dust is: gas stream with dust-loaded from dust generation (1), before gas stream enter in precipitator barrel (3), they mixed fully in duct (2), when gas stream flows through the spray zone which generate by nozzle (5), dust are removed under the action of the wet droplet's interception and inertial collision, next, wet droplet with dust are removed under the rotating fiber apparatus (6) which is driven by motor (4) once more, last the cleaned gas stream is discharge pass by duct (9), the power, which air flow need, is obtained by blower (11).

To evaluate the performance of rotating apparatus, experimental setup, which is shown in Fig.2, is built, and the distribution of experimental dust diameter is given in Fig.3. the relationship of efficiency and pressure loss versus velocity are measured. Results shown in Fig.4, Fig5, respectively.
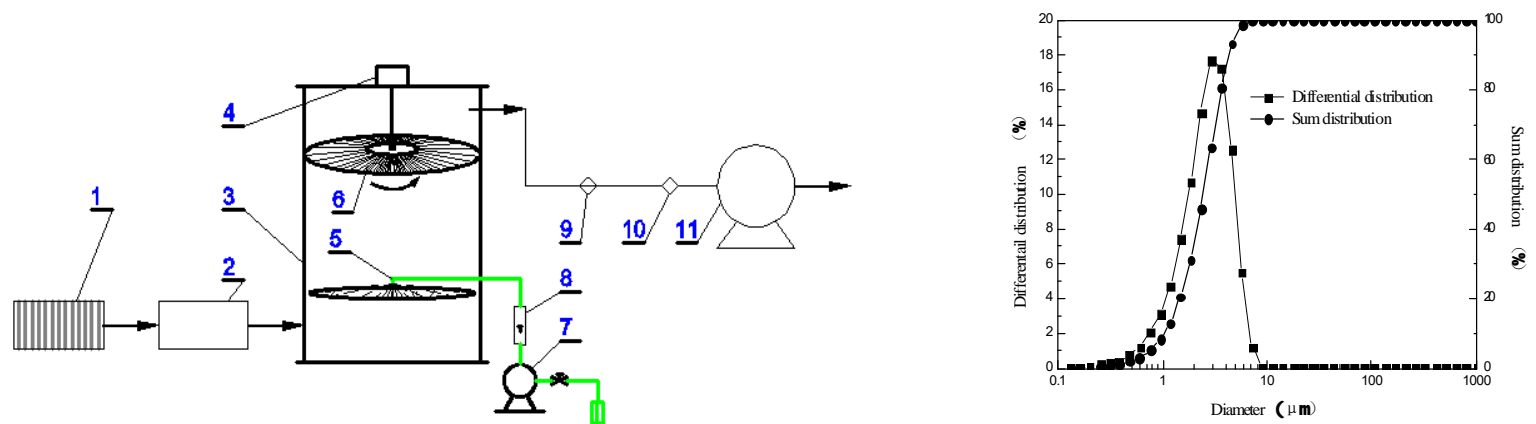

Fig. 2 experimental setup schematic

Fig. 3 distribution of experimental dust diameter 
1 air filter 2 dust generation 3 precipitator barrel 4 motor 5 nozzle 6 rotating fiber apparatus 7 cycle pump 8 flow-meter 9 pressure measured hole 10 measuring hole 11 blower

Given the dust concentration is homogeneous, the mass method is used in efficiency measured, the collection efficiency is calculated according to formula (1)

$$
\eta=1-\frac{c_{2}}{c_{1}}
$$

where, $\eta$ collection efficiency

$$
\begin{aligned}
& \mathrm{c}_{2}-\text { dust concentration in outlet, } \mathrm{mg} / \mathrm{m}^{3} \\
& \mathrm{c}_{1}-\text { dust concentration in inlet, } \mathrm{mg} / \mathrm{m}^{3}
\end{aligned}
$$

Fig. 3 shows the distribution of experimental dust which is limestone dust, the differential distribution and the sum distribution of dust is also shown. The mean diameter is $2.5 \mu \mathrm{m}$.

Fig. 4 shows the relationship of the efficiency versus velocity of the gas and angle velocity of rotating fiber apparatus. it is demonstration that efficiency is decreasing with the velocity of flow gas increasing under angle velocity stead condition. The removal efficiency of fine particles is average 98 percent at $1 \mathrm{~m} / \mathrm{s}$ velocity and at three angle velocity. The removal efficiency of fine particles is above 92 percent. Range the velocity the angle velocity of rotating is less effect for removal of efficiency from results.

Fig. 5 shows the relationship of the pressure drop versus velocity of the gas. The pressure drop is minimum at $1 \mathrm{~m} / \mathrm{s}$ velocity, and it is maximum at $4 \mathrm{~m} / \mathrm{s}$ velocity, range $1-4 \mathrm{~m} / \mathrm{s}$. The maximum pressure drop is not more than $150 \mathrm{~Pa}$.

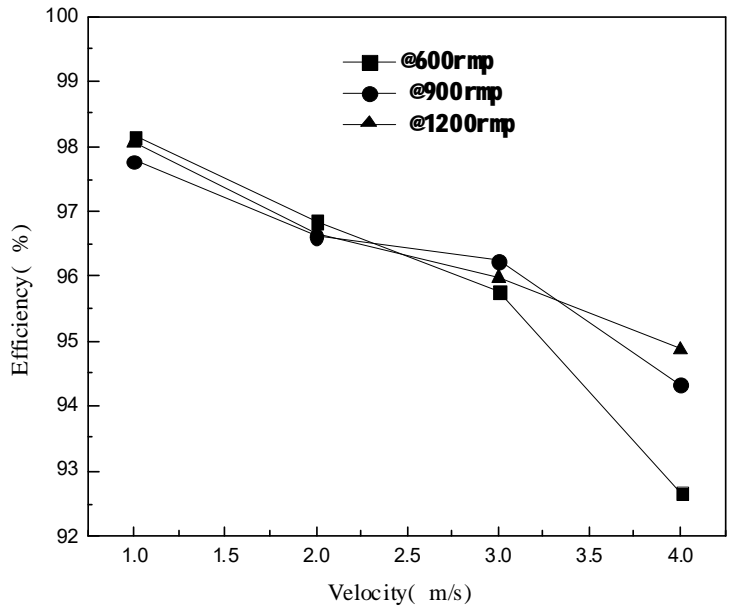

Fig.4 Relationship of efficiency vs. velocity

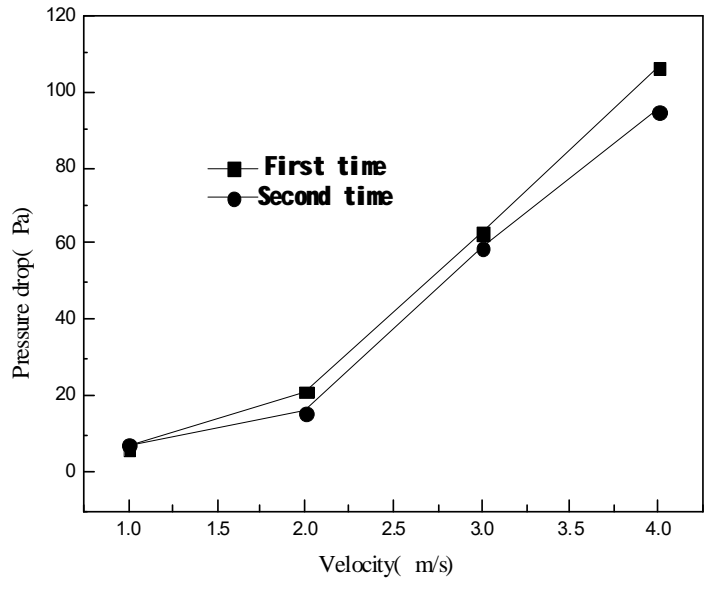

Fig.5 Relationship of pressure vs. velocity

\section{Design and its application testing}

Based on experimental results of the rotating fiber apparatus, the goal of the designed precipitator is the collection efficiency by $80 \%$, the capacity of the air cleaning is at $5000 \mathrm{Nm}^{3}$ air $/ \mathrm{h}$, the pressure drop is less than $500 \mathrm{~Pa}$. According to this goal, A wet precipitator, which the rotating fiber apparatus is served as key part, is designed. The wet dust precipitator designed consists of four parts: the precipitator shell, the rotating fiber apparatus, spray nozzle, collecting water tank, 
referenced [6] detail. It is remarkable characteristics that 4 rotating fiber apparatus are parallel at one section and two are serial by axial line. So there are 8 rotating fiber apparatus used in a wet precipitator. Fig. 6 shows the cross-section of the wet precipitator. It has a length of $850 \mathrm{~mm}$, a width of $503 \mathrm{~mm}$ and a height of $503 \mathrm{~mm}$, respectively. The weight of the wet dust precipitator is 51 kilograms. The typical operating condition is by $10 \mathrm{~m} / \mathrm{s}$ velocity of air flow and the rate of liquid to gas $0.4 \mathrm{~L} / \mathrm{m}^{3}$. All those can also meet the need of mobility and light weight, addition to technique data.

The wet dust precipitator is mounted at the discharging mine platform for application testing under a mine tunnel in Guangxi somewhat mine. Fig.6 demonstrates the testing system. The separation wall 2 is located at middle of fan and the wet dust precipitator, the right side of the separation wall is polluted region by the mineral dust, there is a measuring spot for dust in the front of the fan, and the other measuring spot is located in the rear of the wet dust precipitator. The FC-2 model dust sampler is adopted for testing the concentration of dust, two samplers are mounted in upstream and downstream (demonstrated in Fig.6), respectively. Two samplers are turned on simultaneously after it is regulated to the set volumetric flow rate of air. It takes 2 hours to conduct testing experiment.

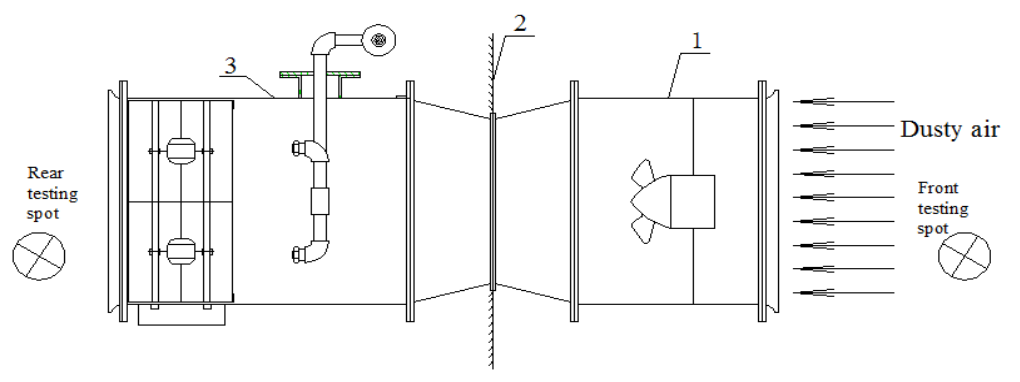

Fig.6 Application testing system of the wet dust precipitator

1 fan 2 separating wall 3 precipitator

According to Determination of dust in the air of workplace-Part 2: Respirable dust concentration (GBZ/ T192.2.2007) and Part 4: Content of free silica in dust (GBZ/ T1 92.4.2007), it take 10 minutes for the wet precipitator application . 10 couple samples are obtained during testing, and the sample is analyzed by Hunan Labor Protection Institute of Nonferrous Metals, the Tab. 3 shows the results of the efficiency is calculated according to the formula (1) .

Table 1 Respirable dust analysis result table

\begin{tabular}{lllllllllll}
\hline No. & 1 & 2 & 3 & 4 & 5 & 6 & 7 & 8 & 9 & 10 \\
\hline Efficiency $\%$ & 32.77 & 96.51 & 92.75 & 94.37 & 92.10 & 88.80 & 99.41 & 54.45 & 87.04 & 64.9
\end{tabular}

It is shown that the minimum removal efficiency of the wet dust precipitator is $32.77 \%$, the maximum $99.41 \%$. The average collection efficiency of the wet precipitator is $80.311 \%$ 。 The maximum respirable dust concentration is at $9.52 \mathrm{mg} / \mathrm{m}^{3}$, the minimum $0.08 \mathrm{mg} / \mathrm{m}^{3}$, the average concentration $2.672 \mathrm{mg} / \mathrm{m}^{3}$ in the outlet of precipitator. Comparing with the result of the single rotating fiber apparatus, the efficiency decreasing is not obvious, though the velocity increasing is great. From the data, there is differential on the dust concentration. The cause might be that the 
measuring is start when the precipitator do not operate steadily, or something is attached on the filter during the installing, dismounting and transporting of filter.

Contrasting to the mine tunnel dust precipitator which is developed by Peiyun $\mathrm{xu}$ and $\mathrm{Li}$ Zhi-bin is at 70 percent collection efficiency ${ }^{[4,5]}$, the wet precipitator operates by removal of efficiency for respirable dust at 80.31 percent averagely, the lift of collection efficiency is obvious, so the application of the wet precipitator will improve the quality of tunnel atmosphere, and it has advantage over decreasing the morbidity of silicosis.

There are some defaults in the wet precipitator such as bringing water into air in the outlet of precipitator due to high velocity, optimizing the frame of the precipitator to decrease its weight.

\section{Conclusions}

The wet precipitator is designed utilizing the rotating fiber apparatus which is served as key part and dehydration part. it is remarkable performance that the pressure drop is low and dust easily deprived from fiber under centrifugal force.

The wet precipitator is suitable for the mine tunnel humidity environment, and its weight is 51 kilograms only, so it is convenient for lifting.

\section{Acknowledgment}

The authors would like to thank Hubei Key Laboratory of Industrial Fume \& Dust Pollution open foundation ( HBIK2017-05 ) and Hubei province natural science foundation ( 2016CF093) for supporting this.

\section{References}

[1] Fred N. Kisselll, Handbook for Dust Control in Mining, Information circulation of NIOSH(2003), 1-132.

[2] C.Y. Chen, Filtration of aerosols by fibrous media, from web

[3] R.Triscori , S.Kumar, Y.Lau, K.Niznik, Air \& Waste Management Association 100th Annual Conference (June 26-29, 2007) Pittsurgh ,Pennsylvania U.S.A.

[4] Qian Peiyun,Xu Qiang,Zhu zhixing etc., Colliery Mechanical \& Electrical Technology (2003) , 5,109-111

[5] Li Zhi-bin, ZHU Chang-yi, LIU Ren-zhi, ZHANG Guo-dong etc., Coal (2007),16 (1), $15-17$

[6] Shi ling, Yang chengwu,Yu xinming, Luo xianwei, Advanced Materials Research, Vol. 800 (2013), pp.8-12 . 